\title{
ON THE PRE-JENSEN-GRÜSS INEQUALITY
}

\section{KLARIČIĆ BAKULA AND J. PEČARIĆ}

\begin{abstract}
Several pre-Jensen-Grüss inequalities for functions of $n$ real variables are proved. The obtained inequalities are refinements of the Jensen-Grüss inequalities proved by Budimir and Pečarić.
\end{abstract}

Mathematics subject classification (2010): 26D15.

Keywords and phrases: Jensen inequality, Grüss inequality, Lipschitz condition, $\alpha$-Hölder condition.

\section{Introduction}

Let $I \subset \mathbb{R}$ be an interval. It is well known that if a function $f: I \rightarrow \mathbb{R}$ is convex then

$$
f\left(\frac{1}{P_{k}} \sum_{i=1}^{k} p_{i} x_{i}\right) \leqslant \frac{1}{P_{k}} \sum_{i=1}^{k} p_{i} f\left(x_{i}\right),
$$

for all $k \in \mathbb{N}, x_{1}, \ldots, x_{k} \in I$ and $p_{1}, \ldots, p_{k} \geqslant 0$ such that $P_{k}=p_{1}+\cdots+p_{k}>0$. If $f$ is strictly convex then (1) is strict unless all $x_{i}$ are equal [5, p. 43]. This is the classical Jensen inequality, one of the most important inequalities in convex analysis, and it has various applications in mathematics, statistics, economics and engineering sciences.

Another important inequality in analysis is the Grüss inequality. It states that

$$
\left|\frac{1}{b-a} \int_{a}^{b} f(s) g(s) \mathrm{d} s-\frac{1}{b-a} \int_{a}^{b} f(s) \mathrm{d} s \cdot \frac{1}{b-a} \int_{a}^{b} g(s) \mathrm{d} s\right| \leqslant \frac{1}{4}(\Gamma-\gamma)(\Phi-\phi)
$$

holds for integrable functions $f, g:[a, b] \rightarrow \mathbb{R}$ such that $\gamma \leqslant f(s) \leqslant \Gamma$, and $\phi \leqslant g(s) \leqslant$ $\Phi$, for all $s \in[a, b]$, where $\gamma, \Gamma, \phi, \Phi \in \mathbb{R}[4$, p. 296].

In [1, Theorem 1] Budimir and Pečarić proved an inequality which they named the Jensen-Grüss inequality: it gives an upper bound for the difference between the right hand side and the left hand side of the Jensen inequality in terms of the Grüss inequality. The goal of this paper is to refine their results under various conditions. It is worth mentioning here that many interesting results of this type can be found in [2] including related results for convex functions [2, pp 191 - 194]. 


\section{Main results}

In the rest of the paper $\operatorname{conv}[S]$ denotes the convex hull of $S \subseteq \mathbb{R}^{n},\langle\cdot, \cdot\rangle: \mathbb{R}^{n} \times$ $\mathbb{R}^{n} \rightarrow \mathbb{R}$ is the inner product on the real $n$-space $\mathbb{R}^{n},\|\cdot\|$ norm related to $\langle\cdot, \cdot\rangle$, and $\leqslant$ the coordinatewise partial order on $\mathbb{R}^{n}$. Also, for the sake of simplicity, for some $k \in \mathbb{N}$ we will often use the notation

$$
P_{k}=p_{1}+\cdots+p_{k}, \quad \bar{x}=\frac{1}{P_{k}} \sum_{i=1}^{k} p_{i} x_{i} .
$$

The next theorem follows the ideas first introduced in [2, Theorem 232, Theorem 237].

THEOREM 1. Let $f: U \rightarrow \mathbb{R}$ be a differentiable function, and $x_{1}, \ldots, x_{k} \in U$, where $U \subset \mathbb{R}^{n}$ is an open convex set. Suppose $m, M \in \mathbb{R}^{n}$ are such that

$$
\langle M-\nabla f(x), \nabla f(x)-m\rangle \geqslant 0 \text { for all } x \in \operatorname{conv}\left[x_{1}, \ldots, x_{k}\right] .
$$

Then for all $p_{i} \geqslant 0, i=1,2, \ldots, k$, such that $P_{k}>0$ the following inequalities hold

$$
\begin{aligned}
& \left|\frac{1}{P_{k}} \sum_{i=1}^{k} p_{i} f\left(x_{i}\right)-f(\bar{x})\right| \\
& \leqslant \frac{1}{2 P_{k}}\|M-m\| \sum_{i=1}^{k} p_{i}\left\|x_{i}-\bar{x}\right\| \leqslant \frac{1}{2}\|M-m\| \tilde{x},
\end{aligned}
$$

where

$$
\tilde{x}=\left(\frac{1}{P_{k}} \sum_{i=1}^{k} p_{i}\left\|x_{i}\right\|^{2}-\|\bar{x}\|^{2}\right)^{\frac{1}{2}} .
$$

Proof. Let $p_{i} \geqslant 0, i=1,2, \ldots, k$, be such that $P_{k}>0$.

From the mean-value theorem we know that for any $x, y \in \operatorname{conv}\left[x_{1}, \ldots, x_{k}\right]$ there exists some $\theta \in(0,1)$ such that

$$
f(y)-f(x)=\langle\nabla f(z), y-x\rangle,
$$

where $z=y+\theta(x-y)$. Applying this on $x=\bar{x} \in \operatorname{conv}\left[x_{1}, \ldots, x_{k}\right], y=x_{i}$ and $z=z_{i}=$ $x_{i}+\theta_{i}\left(\bar{x}-x_{i}\right) \in \operatorname{conv}\left[x_{1}, \ldots, x_{k}\right]$ we obtain

$$
f\left(x_{i}\right)-f(\bar{x})=\left\langle\nabla f\left(z_{i}\right), x_{i}-\bar{x}\right\rangle, i=1,2, \ldots, k .
$$

Multiplying the above equality by $p_{i}$ and summing over $i$ we get

$$
\begin{aligned}
\sum_{i=1}^{k} p_{i} f\left(x_{i}\right)-P_{k} f(\bar{x}) & =\sum_{i=1}^{k} p_{i}\left\langle\nabla f\left(z_{i}\right), x_{i}-\bar{x}\right\rangle \\
& =\sum_{i=1}^{k} p_{i}\left(\left\langle\nabla f\left(z_{i}\right), x_{i}\right\rangle-\left\langle\nabla f\left(z_{i}\right), \bar{x}\right\rangle\right),
\end{aligned}
$$


therefore,

$$
\frac{1}{P_{k}} \sum_{i=1}^{k} p_{i} f\left(x_{i}\right)-f(\bar{x})=\frac{1}{P_{k}} \sum_{i=1}^{k} p_{i}\left\langle\nabla f\left(z_{i}\right), x_{i}\right\rangle-\left\langle\frac{1}{P_{k}} \sum_{i=1}^{k} p_{i} \nabla f\left(z_{i}\right), \bar{x}\right\rangle .
$$

As in the proof of [2, Theorem 232] the right hand side of (4) can be formulated in the following way:

$$
\begin{aligned}
& \frac{1}{P_{k}} \sum_{i=1}^{k} p_{i}\left\langle\nabla f\left(z_{i}\right), x_{i}\right\rangle-\left\langle\frac{1}{P_{k}} \sum_{i=1}^{k} p_{i} \nabla f\left(z_{i}\right), \bar{x}\right\rangle \\
& =\frac{1}{P_{k}} \sum_{i=1}^{k} p_{i}\left\langle\nabla f\left(z_{i}\right)-\frac{M+m}{2}, x_{i}-\bar{x}\right\rangle .
\end{aligned}
$$

By the triangle inequality and then by the Cauchy-Bunyakovsky-Schwarzinequality for inner product spaces we obtain

$$
\begin{aligned}
& \left|\frac{1}{P_{k}} \sum_{i=1}^{k} p_{i}\left\langle\nabla f\left(z_{i}\right), x_{i}\right\rangle-\left\langle\frac{1}{P_{k}} \sum_{i=1}^{k} p_{i} \nabla f\left(z_{i}\right), \bar{x}\right\rangle\right| \\
& \leqslant \frac{1}{P_{k}} \sum_{i=1}^{k} p_{i}\left|\left\langle\nabla f\left(z_{i}\right)-\frac{M+m}{2}, x_{i}-\bar{x}\right\rangle\right| \\
& \leqslant \frac{1}{P_{k}} \sum_{i=1}^{k} p_{i}\left\|\nabla f\left(z_{i}\right)-\frac{M+m}{2}\right\|\left\|x_{i}-\bar{x}\right\| .
\end{aligned}
$$

It can be easily proved (see [2, Lemma 231]) that

$$
\langle M-x, x-m\rangle \geqslant 0
$$

is equivalent to

$$
\left\|x-\frac{M+m}{2}\right\| \leqslant \frac{1}{2}\|M-m\|
$$

for any $x, m, M \in \mathbb{R}^{n}$ since

$$
\langle M-x, x-m\rangle=\frac{1}{4}\|M-m\|^{2}-\left\|x-\frac{M+m}{2}\right\|^{2} .
$$

Combining this with (2) and (5) we obtain

$$
\begin{aligned}
& \left|\frac{1}{P_{k}} \sum_{i=1}^{k} p_{i}\left\langle\nabla f\left(z_{i}\right), x_{i}\right\rangle-\left\langle\frac{1}{P_{k}} \sum_{i=1}^{k} p_{i} \nabla f\left(z_{i}\right), \bar{x}\right\rangle\right| \\
& \leqslant \frac{1}{2 P_{k}}\|M-m\| \sum_{i=1}^{k} p_{i}\left\|x_{i}-\bar{x}\right\|,
\end{aligned}
$$


which with (4) gives the first inequality in (3).

By the Cauchy-Bunyakovsky-Schwarz inequality for positive sequences we know that

$$
\frac{1}{P_{k}} \sum_{i=1}^{k} p_{i}\left\|x_{i}-\bar{x}\right\| \leqslant\left(\frac{1}{P_{k}} \sum_{i=1}^{k} p_{i}\left\|x_{i}-\bar{x}\right\|^{2}\right)^{\frac{1}{2}},
$$

and a simple calculation yields

$$
\begin{aligned}
\frac{1}{P_{k}} \sum_{i=1}^{k} p_{i}\left\|x_{i}-\bar{x}\right\|^{2} & =\frac{1}{P_{k}} \sum_{i=1}^{k} p_{i}\left\|x_{i}\right\|^{2}-\left\|\frac{1}{P_{k}} \sum_{i=1}^{k} p_{i} x_{i}\right\|^{2} \\
& =\frac{1}{P_{k}} \sum_{i=1}^{k} p_{i}\left\|x_{i}\right\|^{2}-\|\bar{x}\|^{2} \geqslant 0 .
\end{aligned}
$$

Observe that (7) ensures that $\tilde{x}$ is well defined.

From (6) and (7) we get

$$
\frac{1}{P_{k}} \sum_{i=1}^{k} p_{i}\left\|x_{i}-\bar{x}\right\| \leqslant \tilde{x},
$$

which proves the second inequality in (3).

Inequalities of the type (3) are called pre-Jensen-Grüss inequalities: they are refinements of their counterpart Jensen-Grüss inequalities in terms of $x$ or alternatively $\tilde{x}$.

In the next theorem we show that Theorem 1 is a refinement of [1, Theorem 1]

THEOREM 2. Let $f: U \rightarrow \mathbb{R}$ be a differentiable function, and $x_{1}, \ldots, x_{k} \in U$, where $U \subset \mathbb{R}^{n}$ is an open convex set. Suppose $m, M \in \mathbb{R}^{n}$ are such that

$$
\langle M-\nabla f(x), \nabla f(x)-m\rangle \geqslant 0 \text { for all } x \in \operatorname{conv}\left[x_{1}, \ldots, x_{k}\right],
$$

and $\phi, \Phi \in \mathbb{R}^{n}$ such that

$$
\left\langle\Phi-x_{i}, x_{i}-\phi\right\rangle \geqslant 0, i=1,2, \ldots, k .
$$

Then for all $p_{i} \geqslant 0, i=1,2, \ldots, k$, such that $P_{k}>0$ the following inequalities hold

$$
\begin{aligned}
& \left|\frac{1}{P_{k}} \sum_{i=1}^{k} p_{i} f\left(x_{i}\right)-f(\bar{x})\right| \\
& \leqslant \frac{1}{2}\|M-m\| \sum_{i=1}^{k} \frac{p_{i}}{P_{k}}\left\|x_{i}-\bar{x}\right\| \\
& \leqslant \frac{1}{2}\|M-m\| \tilde{x} \leqslant \frac{1}{4}\|\Phi-\phi\|\|M-m\|,
\end{aligned}
$$

where $\tilde{x}$ is as in Theorem 1 . 
Proof. The first two inequalities in (8) follow from Theorem 1. Direct calculation gives

$$
\tilde{x}^{2}=\frac{1}{P_{k}} \sum_{i=1}^{k} p_{i}\left\|x_{i}\right\|^{2}-\|\bar{x}\|^{2}=\langle\Phi-\bar{x}, \bar{x}-\phi\rangle-\frac{1}{P_{k}} \sum_{i=1}^{k} p_{i}\left\langle\Phi-x_{i}, x_{i}-\phi\right\rangle,
$$

and by the conditions of this theorem it follows that

$$
\tilde{x}^{2} \leqslant\langle\Phi-\bar{x}, \bar{x}-\phi\rangle .
$$

It is known that the inequality

$$
\langle a, b\rangle \leqslant \frac{1}{4}\|a+b\|^{2}
$$

holds for any $a, b \in \mathbb{R}^{n}$ (with equality iff $a=b$ ). If we choose

$$
a=\Phi-x_{i}, \quad b=x_{i}-\phi
$$

we get

$$
\left\langle\Phi-x_{i}, x_{i}-\phi\right\rangle \leqslant \frac{1}{4}\|\Phi-\phi\|^{2},
$$

hence

$$
\tilde{x} \leqslant \frac{1}{2}\|\Phi-\phi\|
$$

and the last inequality in (8) follows immediately.

A refinement of [1, Theorem 3] is given in the following theorem.

THEOREM 3. Let $f: U \rightarrow \mathbb{R}$ be a differentiable function, and $x_{1}, \ldots, x_{k} \in U$, where $U \subset \mathbb{R}^{n}$ is an open convex set. Let $\phi, \Phi \in \mathbb{R}^{n}$ be such that

$$
\left\langle\Phi-x_{i}, x_{j}-\phi\right\rangle \geqslant 0, i, j=1,2, \ldots, k \text {. }
$$

Suppose that for some $L>0 \nabla f$ satisfies the Lipschitz condition

$$
\|\nabla f(y)-\nabla f(x)\| \leqslant L\|y-x\| \text { for all } x, y \in \operatorname{conv}\left[x_{1}, \ldots, x_{k}\right] .
$$

Then for all $p_{i} \geqslant 0, i=1,2, \ldots, k$, such that $P_{k}>0$ the following inequalities hold

$$
\left|\frac{1}{P_{k}} \sum_{i=1}^{k} p_{i} f\left(x_{i}\right)-f(\bar{x})\right| \leqslant \frac{L}{2} \tilde{x}\|\Phi-\phi\| \leqslant \frac{L}{4}\|\Phi-\phi\|^{2},
$$

where $\tilde{x}$ is as in Theorem 1.

Proof. We start from (4) obtained as in the proof of Theorem 1. It can be easily proved that

$$
\begin{aligned}
& \frac{1}{P_{k}} \sum_{i=1}^{k} p_{i}\left\langle\nabla f\left(z_{i}\right), x_{i}\right\rangle-\left\langle\frac{1}{P_{k}} \sum_{i=1}^{k} p_{i} \nabla f\left(z_{i}\right), \bar{x}\right\rangle \\
& =\frac{1}{2 P_{k}^{2}} \sum_{i, j=1}^{k} p_{i} p_{j}\left\langle\nabla f\left(z_{i}\right)-\nabla f\left(z_{j}\right), x_{i}-x_{j}\right\rangle .
\end{aligned}
$$


By the Cauchy-Bunyakovsky-Schwarz inequality for inner product spaces from the above equality we get

$$
\begin{aligned}
& \left|\frac{1}{P_{k}} \sum_{i=1}^{k} p_{i}\left\langle\nabla f\left(z_{i}\right), x_{i}\right\rangle-\left\langle\frac{1}{P_{k}} \sum_{i=1}^{k} p_{i} \nabla f\left(z_{i}\right), \bar{x}\right\rangle\right| \\
& \leqslant \frac{1}{2 P_{k}^{2}} \sum_{i, j=1}^{k} p_{i} p_{j}\left|\left\langle\nabla f\left(z_{i}\right)-\nabla f\left(z_{j}\right), x_{i}-x_{j}\right\rangle\right| \\
& \leqslant \frac{1}{2 P_{k}^{2}} \sum_{i, j=1}^{k} p_{i} p_{j}\left\|\nabla f\left(z_{i}\right)-\nabla f\left(z_{j}\right)\right\|\left\|x_{i}-x_{j}\right\| .
\end{aligned}
$$

By the Cauchy-Bunyakovsky-Schwarz inequality for double sums we know that

$$
\begin{aligned}
& \frac{1}{2 P_{k}^{2}} \sum_{i, j=1}^{k} p_{i} p_{j}\left\|x_{i}-x_{j}\right\|\left\|\nabla f\left(z_{i}\right)-\nabla f\left(z_{j}\right)\right\| \\
& \leqslant\left(\frac{1}{2 P_{k}^{2}} \sum_{i, j=1}^{k} p_{i} p_{j}\left\|x_{i}-x_{j}\right\|^{2}\right)^{\frac{1}{2}}\left(\frac{1}{2 P_{k}^{2}} \sum_{i, j=1}^{k} p_{i} p_{j}\left\|\nabla f\left(z_{i}\right)-\nabla f\left(z_{j}\right)\right\|^{2}\right)^{\frac{1}{2}} .
\end{aligned}
$$

A short calculation yields

$$
\frac{1}{2 P_{k}^{2}} \sum_{i, j=1}^{k} p_{i} p_{j}\left\|x_{i}-x_{j}\right\|^{2}=\frac{1}{P_{k}} \sum_{i=1}^{k} p_{i}\left\|x_{i}\right\|^{2}-\|\bar{x}\|^{2},
$$

and analogously

$$
\frac{1}{2 P_{k}^{2}} \sum_{i, j=1}^{k} p_{i} p_{j}\left\|\nabla f\left(z_{i}\right)-\nabla f\left(z_{j}\right)\right\|^{2}=\frac{1}{P_{k}} \sum_{i=1}^{k} p_{i}\left\|\nabla f\left(z_{i}\right)\right\|^{2}-\left\|\frac{1}{P_{k}} \sum_{i=1}^{k} p_{i} \nabla f\left(z_{i}\right)\right\|^{2} .
$$

Now we have

$$
\begin{aligned}
& \left|\frac{1}{P_{k}} \sum_{i=1}^{k} p_{i} f\left(x_{i}\right)-f(\bar{x})\right| \\
& \leqslant\left(\frac{1}{2 P_{k}^{2}} \sum_{i, j=1}^{k} p_{i} p_{j}\left\|x_{i}-x_{j}\right\|^{2}\right)^{\frac{1}{2}}\left(\frac{1}{2 P_{k}^{2}} \sum_{i, j=1}^{k} p_{i} p_{j}\left\|\nabla f\left(z_{i}\right)-\nabla f\left(z_{j}\right)\right\|^{2}\right)^{\frac{1}{2}} \\
& \leqslant\left(\frac{1}{2 P_{k}^{2}} \sum_{i, j=1}^{k} p_{i} p_{j}\left\|x_{i}-x_{j}\right\|^{2}\right)^{\frac{1}{2}}\left(\frac{1}{2 P_{k}^{2}} \sum_{i, j=1}^{k} p_{i} p_{j} L^{2}\left\|z_{i}-z_{j}\right\|^{2}\right)^{\frac{1}{2}} \\
& =L\left(\frac{1}{P_{k}} \sum_{i=1}^{k} p_{i}\left\|x_{i}\right\|^{2}-\|\bar{x}\|^{2}\right)^{\frac{1}{2}}\left(\frac{1}{P_{k}} \sum_{i=1}^{k} p_{i}\left\|z_{i}\right\|^{2}-\|\bar{z}\|^{2}\right)^{\frac{1}{2}} .
\end{aligned}
$$


For any $z \in \operatorname{conv}\left[x_{1}, \ldots, x_{k}\right]$ there are some $u_{i} \in[0,1], i=1,2, \ldots, k$, such that $\sum_{i=1}^{k} u_{i}=$ 1 and $z=\sum_{i=1}^{k} u_{i} x_{i}$. Then

$$
\begin{aligned}
\langle\Phi-z, z-\phi\rangle & =\left\langle\sum_{i=1}^{k} u_{i} \Phi-\sum_{i=1}^{k} u_{i} x_{i}, \sum_{i=1}^{k} u_{i} x_{i}-\sum_{i=1}^{k} u_{i} \phi\right\rangle \\
& =\left\langle\sum_{i=1}^{k} u_{i}\left(\Phi-x_{i}\right), \sum_{i=1}^{k} u_{i}\left(x_{i}-\phi\right)\right\rangle \geqslant 0
\end{aligned}
$$

hence

$$
\left\langle\Phi-z_{i}, z_{i}-\phi\right\rangle \geqslant 0, i=1,2, \ldots, k
$$

This allow us to conclude

$$
\frac{1}{P_{k}} \sum_{i=1}^{k} p_{i}\left\|z_{i}\right\|^{2}-\|\bar{z}\|^{2} \leqslant \frac{1}{4}\|\Phi-\phi\|^{2},
$$

and accordingly

$$
\left|\frac{1}{P_{k}} \sum_{i=1}^{k} p_{i} f\left(x_{i}\right)-f(\bar{x})\right| \leqslant \frac{L}{2} \tilde{x}\|\Phi-\phi\| .
$$

Taking into account (9) we obtain the second inequality in (10) and the proof is complete.

A more general variant of the result given in Theorem 3 , a refinement of [1, Theorem 2], is stated below.

THEOREM 4. Let $f: U \rightarrow \mathbb{R}$ be a differentiable function, and $x_{1}, \ldots, x_{k} \in U$, where $U \subset \mathbb{R}^{n}$ is an open convex set. Suppose that for some $C>0$ and $\alpha \in(0,1) \nabla f$ satisfies the Hölder condition

$$
\|\nabla f(y)-\nabla f(x)\| \leqslant C\|y-x\|^{\alpha} \text { for all } x, y \in \operatorname{conv}\left[x_{1}, \ldots, x_{k}\right] .
$$

Then for all $p_{i} \geqslant 0, i=1,2, \ldots, k$, such that $P_{k}>0$ the following inequalities hold

$$
\begin{aligned}
& \left|\frac{1}{P_{k}} \sum_{i=1}^{k} p_{i} f\left(x_{i}\right)-f(\bar{x})\right| \\
& \leqslant \frac{C \Delta^{\alpha}}{P_{k}^{2}} \sum_{i<j} p_{i} p_{j}\left\|x_{i}-x_{j}\right\| \leqslant \frac{C \Delta^{\alpha}}{P_{k}}\left(\frac{P_{k}^{2}-\sum_{i=1}^{k} p_{i}^{2}}{2}\right)^{\frac{1}{2}} \tilde{x} \\
& \leqslant \frac{C \Delta^{\alpha+1}}{2 P_{k}^{2}}\left(P_{k}^{2}-\sum_{i=1}^{k} p_{i}^{2}\right)
\end{aligned}
$$

where

$$
\Delta=\max _{1 \leqslant i<j \leqslant k}\left\|x_{i}-x_{j}\right\|
$$

and $\tilde{x}$ is as in Theorem 1. 
Proof. First observe that for any $a, b \in \operatorname{conv}\left[x_{1}, \ldots, x_{k}\right]$ there exist some $u_{l}, v_{l} \in$ $[0,1], l=1,2, \ldots, k$, such that $\sum_{l=1}^{k} u_{l}=\sum_{l=1}^{k} v_{l}=1$ and

$$
a=\sum_{l=1}^{k} u_{l} x_{l}, \quad b=\sum_{l=1}^{k} v_{l} x_{l} .
$$

Then

$$
\begin{aligned}
\|a-b\| & =\left\|\sum_{l=1}^{k} v_{l} \sum_{l=1}^{k} u_{l} x_{l}-\sum_{l=1}^{k} u_{l} \sum_{l=1}^{k} v_{l} x_{l}\right\| \\
& =\left\|\sum_{i, j=1}^{k} u_{i} v_{j}\left(x_{i}-x_{j}\right)\right\| \\
& \leqslant \sum_{i, j=1}^{k} u_{i} v_{j}\left\|x_{i}-x_{j}\right\| \leqslant \Delta \sum_{i, j=1}^{k} u_{i} v_{j}=\Delta .
\end{aligned}
$$

Consequently, for $z_{i}, i=1,2, \ldots, k$, defined as in Theorem 1 we have

$$
\left\|z_{i}-z_{j}\right\| \leqslant \Delta, \quad i, j=1,2, \ldots, k
$$

hence

$$
\left\|\nabla f\left(z_{i}\right)-\nabla f\left(z_{j}\right)\right\| \leqslant C\left\|z_{i}-z_{j}\right\|^{\alpha} \leqslant C \Delta^{\alpha}, i, j=1,2, \ldots, k .
$$

From (4) and (11) we get

$$
\begin{aligned}
& \left|\frac{1}{P_{k}} \sum_{j=1}^{k} p_{j} f\left(x_{j}\right)-f(\bar{x})\right| \\
& \leqslant \frac{1}{2 P_{k}^{2}} \sum_{i, j=1}^{k} p_{i} p_{j}\left\|x_{i}-x_{j}\right\|\left\|\nabla f\left(z_{i}\right)-\nabla f\left(z_{j}\right)\right\| \\
& =\frac{1}{P_{k}^{2}} \sum_{i<j} p_{i} p_{j}\left\|x_{i}-x_{j}\right\|\left\|\nabla f\left(z_{i}\right)-\nabla f\left(z_{j}\right)\right\|,
\end{aligned}
$$

hence

$$
\begin{aligned}
& \left|\frac{1}{P_{k}} \sum_{j=1}^{k} p_{j} f\left(x_{j}\right)-f(\bar{x})\right| \\
& \leqslant \frac{C}{P_{k}^{2}} \sum_{i<j} p_{i} p_{j}\left\|x_{i}-x_{j}\right\|\left\|z_{i}-z_{j}\right\|^{\alpha} \\
& \leqslant \frac{C \Delta^{\alpha}}{P_{k}^{2}} \sum_{i<j} p_{i} p_{j}\left\|x_{i}-x_{j}\right\|,
\end{aligned}
$$


which is the first inequality in (13). Applying the comparison inequality between the arithmetic and quadratic mean and (12) we obtain

$$
\begin{aligned}
& \frac{2}{P_{k}^{2}-\sum_{i=1}^{k} p_{i}^{2}} \sum_{i<j} p_{i} p_{j}\left\|x_{i}-x_{j}\right\| \\
& \leqslant\left(\frac{2}{P_{k}^{2}-\sum_{i=1}^{k} p_{i}^{2}} \sum_{i<j} p_{i} p_{j}\left\|x_{i}-x_{j}\right\|^{2}\right)^{\frac{1}{2}} \\
& =\left(\frac{2 P_{k}^{2}}{P_{k}^{2}-\sum_{i=1}^{k} p_{i}^{2}}\right)^{\frac{1}{2}}\left(\frac{1}{2 P_{k}^{2}} \sum_{i, j=1}^{k} p_{i} p_{j}\left\|x_{i}-x_{j}\right\|^{2}\right)^{\frac{1}{2}} \\
& =\left(\frac{2 P_{k}^{2}}{P_{k}^{2}-\sum_{i=1}^{k} p_{i}^{2}}\right)^{\frac{1}{2}}\left(\frac{1}{P_{k}} \sum_{i=1}^{k} p_{i}\left\|x_{i}\right\|^{2}-\|\bar{x}\|^{2}\right)^{\frac{1}{2}} \\
& =\left(\frac{2 P_{k}^{2}}{P_{k}^{2}-\sum_{i=1}^{k} p_{i}^{2}}\right)^{\frac{1}{2}} \tilde{x}
\end{aligned}
$$

hence

$$
\begin{aligned}
\sum_{i<j} p_{i} p_{j}\left\|x_{i}-x_{j}\right\| & \leqslant\left(\frac{P_{k}^{2}-\sum_{i=1}^{k} p_{i}^{2}}{2}\right)^{\frac{1}{2}}\left(\sum_{i<j} p_{i} p_{j}\left\|x_{i}-x_{j}\right\|^{2}\right)^{\frac{1}{2}} \\
& \leqslant \Delta\left(\frac{P_{k}^{2}-\sum_{i=1}^{k} p_{i}^{2}}{2}\right)^{\frac{1}{2}}\left(\sum_{i<j} p_{i} p_{j}\right)^{\frac{1}{2}} \\
& =\frac{\Delta}{2}\left(P_{k}^{2}-\sum_{i=1}^{k} p_{i}^{2}\right)
\end{aligned}
$$

which proves the second and the third inequality in (13).

\section{One dimensional case}

In this section we consider functions defined on $I=(a, b) \subseteq \mathbb{R}, a<b$.

THEOREM 5. Let $f: I \rightarrow \mathbb{R}$ be a differentiable function, and $x_{1}, \ldots, x_{k} \in I$. Suppose $m, M \in \mathbb{R}$ are such that

$$
m \leqslant f^{\prime}(x) \leqslant M, \text { for all } x \in I .
$$


Then for all $p_{i} \geqslant 0, i=1,2, \ldots, k$, such that $P_{k}>0$ the following inequalities hold

$$
\begin{aligned}
& \left|\frac{1}{P_{k}} \sum_{i=1}^{k} p_{i} f\left(x_{i}\right)-f(\bar{x})\right| \\
& \leqslant \frac{M-m}{2 P_{k}} \sum_{i=1}^{k} p_{i}\left|x_{i}-\bar{x}\right| \\
& \leqslant \frac{M-m}{2 P_{k}^{2}} \sum_{i=1}^{k} p_{i} \sum_{j=1}^{k} p_{j}\left|x_{i}-x_{j}\right| \\
& \leqslant \frac{(M-m)(b-a)}{P_{k}^{2}} \max _{J \subset\{1, \ldots, k\}} P(J)\left(P_{k}-P(J)\right),
\end{aligned}
$$

where $P(J)=\sum_{i \in J} p_{i}$.

Proof. For $n=1$ identity (4) becomes

$$
\frac{1}{P_{k}} \sum_{i=1}^{k} p_{i} f\left(x_{i}\right)-f(\bar{x})=\frac{1}{P_{k}} \sum_{i=1}^{k} p_{i} x_{i} f^{\prime}\left(z_{i}\right)-\bar{x} \frac{1}{P_{k}} \sum_{i=1}^{k} p_{i} f^{\prime}\left(z_{i}\right),
$$

hence

$$
\begin{aligned}
& \left|\frac{1}{P_{k}} \sum_{i=1}^{k} p_{i} f\left(x_{i}\right)-f(\bar{x})\right| \\
& =\left|\frac{1}{P_{k}} \sum_{i=1}^{k} p_{i} x_{i} f^{\prime}\left(z_{i}\right)-\frac{1}{P_{k}^{2}} \sum_{i=1}^{k} p_{i} x_{i} \sum_{i=1}^{k} p_{i} f^{\prime}\left(z_{i}\right)\right|
\end{aligned}
$$

In [6, Theorem 2.4] it was proved that for any $w_{i} \in \mathbb{R}, i=1,2, \ldots, k$, such that $W_{k}=1$, and any $a_{i}, b_{i}, i=1,2, \ldots, k$, such that $m_{1}<a_{i}<M_{1}$ and $m_{2}<b_{i}<M_{2}, i=1,2, \ldots, k$, the following inequalities hold

$$
\begin{aligned}
& \left|\sum_{i=1}^{k} w_{i} a_{i} b_{i}-\sum_{i=1}^{k} w_{i} a_{i} \sum_{i=1}^{k} w_{i} b_{i}\right| \\
& \leqslant \frac{M_{2}-m_{2}}{2} \sum_{i=1}^{k} w_{i}\left|a_{i}-\sum_{i=1}^{k} w_{i} a_{i}\right| \\
& \leqslant \frac{M_{2}-m_{2}}{2} \sum_{i=1}^{k} w_{i} \sum_{j=1}^{k} w_{j}\left|a_{i}-a_{j}\right| \\
& \leqslant\left(M_{1}-m_{1}\right)\left(M_{2}-m_{2}\right) \max _{J \subset\{1, \ldots, k\}} W(J)(1-W(J)),
\end{aligned}
$$


where $W(J)=\sum_{i \in J} w_{i}$. If we apply (16) on the right hand side of (15) choosing $w_{i}=p_{i} / P_{k}, a_{i}=x_{i}$ and $b_{i}=f^{\prime}\left(z_{i}\right)$ we obtain

$$
\begin{aligned}
\left|\frac{1}{P_{k}} \sum_{i=1}^{k} p_{i} f\left(x_{i}\right)-f(\bar{x})\right| & \leqslant \frac{M-m}{2 P_{k}} \sum_{i=1}^{k} p_{i}\left|x_{i}-\bar{x}\right| \\
& \leqslant \frac{M-m}{2 P_{k}^{2}} \sum_{i=1}^{k} p_{i} \sum_{j=1}^{k} p_{j}\left|x_{i}-x_{j}\right| \\
& \leqslant \frac{(M-m)(b-a)}{P_{k}^{2}} \max _{J \subset\{1, \ldots, k\}} P(J)\left(P_{k}-P(J)\right) .
\end{aligned}
$$

This completes the proof.

REMARK 1. In [6, Theorem 2.4] it was originally proved that (16) holds for any $w_{i} \in \mathbb{R}, i=1,2, \ldots, k$, such that $W_{k}=1$ and $a_{i}, b_{i}, i=1,2, \ldots, k$, such that $0<m_{1}<$ $a_{i}<M_{1}$ and $0<m_{2}<b_{i}<M_{2}, i=1,2, \ldots, k$. But it can be easily seen that the conditions $0<m_{1}$ and $0<m_{2}$ are not essential, we can simply choose two new strings $a_{i}^{\prime}=M_{1}-a_{i}$ and $b_{i}^{\prime}=M_{2}-b_{i}$ to obtain the same result.

REMARK 2. There is an alternative way to refine [1, Theorem 4] under the same conditions in terms of $\tilde{x}$ as it is shown below. We start as in Theorem 5 but instead of proceeding with the whole string of inequalities given in [6, Theorem 2.4] we use only the first two and then the comparison inequality between the arithmetic and quadratic mean.

$$
\begin{aligned}
& \left|\frac{1}{P_{k}} \sum_{i=1}^{k} p_{i} f\left(x_{i}\right)-f(\bar{x})\right| \\
& \leqslant \frac{M-m}{2 P_{k}} \sum_{i=1}^{k} p_{i}\left|x_{i}-\bar{x}\right| \leqslant \frac{M-m}{P_{k}^{2}} \sum_{i<j} p_{i} p_{j}\left|x_{i}-x_{j}\right| \\
& =\frac{M-m}{P_{k}^{2}} \frac{P_{k}^{2}-\sum_{i=1}^{k} p_{i}^{2}}{2}\left(\frac{2}{P_{k}^{2}-\sum_{i=1}^{k} p_{i}^{2}} \sum_{i<j} p_{i} p_{j}\left|x_{i}-x_{j}\right|\right) \\
& \leqslant \frac{M-m}{P_{k}^{2}} \frac{P_{k}^{2}-\sum_{i=1}^{k} p_{i}^{2}}{2}\left(\frac{2}{P_{k}^{2}-\sum_{i=1}^{k} p_{i}^{2}} \sum_{i<j} p_{i} p_{j}\left|x_{i}-x_{j}\right|^{2}\right)^{\frac{1}{2}} \\
& =\frac{M-m}{P_{k}}\left(\frac{P_{k}^{2}-\sum_{i=1}^{k} p_{i}^{2}}{2}\right)^{\frac{1}{2}}\left(\frac{1}{P_{k}^{2}} \sum_{i<j} p_{i} p_{j}\left|x_{i}-x_{j}\right|^{2}\right)^{\frac{1}{2}} .
\end{aligned}
$$

We already know that

$$
\tilde{x}^{2}=\frac{1}{P_{k}^{2}} \sum_{i<j} p_{i} p_{j}\left|x_{i}-x_{j}\right|^{2},
$$


hence

$$
\begin{aligned}
& \left|\frac{1}{P_{k}} \sum_{i=1}^{k} p_{i} f\left(x_{i}\right)-f(\bar{x})\right| \\
& \leqslant \frac{M-m}{2 P_{k}} \sum_{i=1}^{k} p_{i}\left|x_{i}-\bar{x}\right| \leqslant \frac{M-m}{P_{k}^{2}} \sum_{i<j} p_{i} p_{j}\left|x_{i}-x_{j}\right| \\
& \leqslant \frac{M-m}{P_{k}}\left(\frac{P_{k}^{2}-\sum_{i=1}^{k} p_{i}^{2}}{2}\right)^{\frac{1}{2}} \tilde{x} .
\end{aligned}
$$

In [3, Corollary 2.5] the authors proved, under the same conditions as in [6, Theorem 2.4], that the following inequality holds

$$
\sum_{i<j} w_{i} w_{j}\left(a_{i}-a_{j}\right)^{2} \leqslant(M-m)^{2} \max _{J \subset\{1, \ldots, k\}} W(J)(1-W(J)) .
$$

If we apply it choosing $w_{i}=p_{i} / P_{k}$ and $a_{i}=x_{i}$ we get

$$
\frac{1}{P_{k}^{2}} \sum_{i<j} p_{i} p_{j}\left|x_{i}-x_{j}\right|^{2} \leqslant(b-a)^{2} \max _{J \subset\{1, \ldots, k\}} \frac{P(J)\left(P_{k}-P(J)\right)}{P_{k}^{2}},
$$

hence

$$
\begin{aligned}
& \left|\frac{1}{P_{k}} \sum_{i=1}^{k} p_{i} f\left(x_{i}\right)-f(\bar{x})\right| \\
& \leqslant \frac{M-m}{2 P_{k}} \sum_{i=1}^{k} p_{i}\left|x_{i}-\bar{x}\right| \leqslant \frac{M-m}{P_{k}^{2}} \sum_{i<j} p_{i} p_{j}\left|x_{i}-x_{j}\right| \\
& \leqslant \frac{M-m}{P_{k}}\left(\frac{P_{k}^{2}-\sum_{i=1}^{k} p_{i}^{2}}{2}\right)^{\frac{1}{2}} \tilde{x} \\
& =\frac{M-m}{P_{k}}\left(\frac{P_{k}^{2}-\sum_{i=1}^{k} p_{i}^{2}}{2}\right)^{\frac{1}{2}}\left(\frac{1}{P_{k}^{2}} \sum_{i<j} p_{i} p_{j}\left|x_{i}-x_{j}\right|^{2}\right)^{\frac{1}{2}} \\
& \leqslant \frac{(M-m)(b-a)}{P_{k}^{2}}\left(\frac{P_{k}^{2}-\sum_{i=1}^{k} p_{i}^{2}}{2}\right)^{\frac{1}{2}}\left(\max _{J \subset\{1, \ldots, k\}} P(J)\left(P_{k}-P(J)\right)\right)^{\frac{1}{2}} .
\end{aligned}
$$

Note that by a similar argument as in

$$
\langle a, b\rangle \leqslant \frac{1}{4}\|a+b\|^{2}
$$

we can prove

$$
\frac{1}{P_{k}}\left(\frac{P_{k}^{2}-\sum_{i=1}^{k} p_{i}^{2}}{2}\right)^{\frac{1}{2}} \leqslant \frac{1}{2}
$$


and consequently

$$
\frac{M-m}{P_{k}}\left(\frac{P_{k}^{2}-\sum_{i=1}^{k} p_{i}^{2}}{2}\right)^{\frac{1}{2}} \tilde{x} \leqslant \frac{M-m}{2} \tilde{x} .
$$

THEOREM 6. Let $f: I \rightarrow \mathbb{R}$ be a differentiable function, and $x_{1}, \ldots, x_{k} \in I$. Suppose that for some $L>0 f^{\prime}$ satisfies the Lipschitz condition

$$
\left|f^{\prime}(y)-f^{\prime}(x)\right| \leqslant L|y-x| \text { for all } x, y \in I \text {. }
$$

Then for all $p_{i} \geqslant 0, i=1,2, \ldots, k$, such that $P_{k}>0$ the following inequalities hold

$$
\begin{aligned}
& \left|\frac{1}{P_{k}} \sum_{i=1}^{k} p_{i} f\left(x_{i}\right)-f(\bar{x})\right| \leqslant L \frac{b-a}{P_{k}^{2}} \sum_{i<j} p_{i} p_{j}\left|x_{i}-x_{j}\right| \\
& \leqslant L \frac{(b-a)^{2}}{P_{k}^{2}} \max _{J \subset\{1, \ldots, k\}} P(J)\left(P_{k}-P(J)\right) .
\end{aligned}
$$

Proof. For $n=1$ the first inequality in (11) becomes

$$
\left|\frac{1}{P_{k}} \sum_{i=1}^{k} p_{i} f\left(x_{i}\right)-f(\bar{x})\right| \leqslant \frac{1}{2 P_{k}^{2}} \sum_{i, j=1}^{k} p_{i} p_{j}\left|x_{i}-x_{j}\right|\left|f^{\prime}\left(z_{i}\right)-f^{\prime}\left(z_{j}\right)\right| .
$$

Since $f^{\prime}$ is Lipschitzian on $I$ we may conclude

$$
\begin{aligned}
\left|\frac{1}{P_{k}} \sum_{i=1}^{k} p_{i} f\left(x_{i}\right)-f(\bar{x})\right| & \leqslant \frac{L}{2 P_{k}^{2}} \sum_{i, j=1}^{k} p_{i} p_{j}\left|x_{i}-x_{j}\right|\left|z_{i}-z_{j}\right| \\
& =\frac{L}{P_{k}^{2}} \sum_{i<j} p_{i} p_{j}\left|x_{i}-x_{j}\right|\left|z_{i}-z_{j}\right| \\
& \leqslant L \frac{b-a}{P_{k}^{2}} \sum_{i<j} p_{i} p_{j}\left|x_{i}-x_{j}\right| .
\end{aligned}
$$

In [3, Corollary 2.4] the authors proved, under the same conditions as it was mentioned before, that the following inequality holds

$$
\sum_{i<j} w_{i} w_{j}\left|a_{i}-a_{j}\right| \leqslant(M-m) \max _{J \subset\{1, \ldots, k\}} W(J)(1-W(J)) .
$$

If we apply (18) on the right hand side of (17) choosing $w_{i}=p_{i} / P_{k}, a_{i}=x_{i}$, we obtain

$$
\begin{aligned}
& \left|\frac{1}{P_{k}} \sum_{i=1}^{k} p_{i} f\left(x_{i}\right)-f(\bar{x})\right| \leqslant L \frac{b-a}{P_{k}^{2}} \sum_{i<j} p_{i} p_{j}\left|x_{i}-x_{j}\right| \\
& \leqslant L \frac{(b-a)^{2}}{P_{k}^{2}} \max _{J \subset\{1, \ldots, k\}} P(J)\left(P_{k}-P(J)\right),
\end{aligned}
$$

and this concludes the proof. 
REMARK 3. We can obtain a similar upper bound as in Remark 2 if we start as in Theorem 6 but instead of using [3, Corollary 2.4] use (as in the proof of Theorem 4) the following inequality

$$
\sum_{i<j} p_{i} p_{j}\left|x_{i}-x_{j}\right| \leqslant\left(\frac{P_{k}^{2}-\sum_{i=1}^{k} p_{i}^{2}}{2}\right)^{\frac{1}{2}} P_{k} \tilde{x} .
$$

That way we get

$$
\begin{aligned}
& \left|\frac{1}{P_{k}} \sum_{i=1}^{k} p_{i} f\left(x_{i}\right)-f(\bar{x})\right| \leqslant L \frac{b-a}{P_{k}^{2}} \sum_{i<j} p_{i} p_{j}\left|x_{i}-x_{j}\right| \\
& \leqslant L \frac{b-a}{P_{k}}\left(\frac{P_{k}^{2}-\sum_{i=1}^{k} p_{i}^{2}}{2}\right)^{\frac{1}{2}} \tilde{x} .
\end{aligned}
$$

Now, using again [6, Theorem 2.4] as in Remark 2,

$$
\begin{aligned}
& \left|\frac{1}{P_{k}} \sum_{i=1}^{k} p_{i} f\left(x_{i}\right)-f(\bar{x})\right| \\
& \leqslant L \frac{b-a}{P_{k}^{2}} \sum_{i<j} p_{i} p_{j}\left|x_{i}-x_{j}\right| \leqslant L \frac{b-a}{P_{k}}\left(\frac{P_{k}^{2}-\sum_{i=1}^{k} p_{i}^{2}}{2}\right)^{\frac{1}{2}} \tilde{x} \\
& \leqslant L \frac{(b-a)^{2}}{P_{k}^{2}}\left(\frac{P_{k}^{2}-\sum_{i=1}^{k} p_{i}^{2}}{2}\right)^{\frac{1}{2}}\left(\max _{J \subset\{1, \ldots, k\}} P(J)\left(P_{k}-P(J)\right)\right)^{\frac{1}{2}} .
\end{aligned}
$$

Let us consider Theorem 1 for $n=1$. In that case $\operatorname{conv}\left[x_{1}, \ldots, x_{k}\right]=\left[x_{\min }, x_{\max }\right]$, where $x_{\min }=\min \left\{x_{1}, \ldots, x_{k}\right\}$ and $x_{\max }=\max \left\{x_{1}, \ldots, x_{k}\right\}$, and we suppose $m, M \in \mathbb{R}$ are such that $m \leqslant f^{\prime}(x) \leqslant M$ for all $x \in\left[x_{\min }, x_{\max }\right]$. On the other hand, the condition on $\nabla f$ for $n=1$ becomes

$$
\left(M-f^{\prime}(x)\right)\left(f^{\prime}(x)-m\right) \geqslant 0 \text { for all } x \in\left[x_{\min }, x_{\max }\right],
$$

which is obviously fulfilled if $m \leqslant f^{\prime}(x) \leqslant M$. The inequalities in (3) become

$$
\left|\frac{1}{P_{k}} \sum_{i=1}^{k} p_{i} f\left(x_{i}\right)-f(\bar{x})\right| \leqslant \frac{M-m}{2 P_{k}} \sum_{i=1}^{k} p_{i}\left|x_{i}-\bar{x}\right| \leqslant \frac{M-m}{2} \tilde{x},
$$

and we can see that the first inequality in (14) is the same. In Remark 2 we proved that the second inequality in (14) is better than the second one obtained in Theorem 1 when $n=1$.

Theorem 3 for $n=1$ states

$$
\left|\frac{1}{P_{k}} \sum_{i=1}^{k} p_{i} f\left(x_{i}\right)-f(\bar{x})\right| \leqslant L \frac{b-a}{2} \tilde{x}
$$


and by a similar argument as before we may conclude that Theorem 6 provides better bound than Theorem 3 for this simplest choice of $\Phi$ and $\phi$.

Acknowledgement. The authors are very grateful to the referee for many constructive comments and recommendations which improved the readability and quality of the paper.

\section{REFERENCES}

[1] I. Budimir, J. PeČArić, The Jensen-Grüss inequality, Math. Inequal. Appl. 5 (2002), no. 2, 205214.

[2] Sever Silvestru Dragomir, Advances in inequalities of the Schwarz, Grüss and Bessel type in inner product spaces, Nova Science Publishers, Inc., Hauppauge, NY, 2005.

[3] Saichi Izumino, Josip E. PeČArić, Some extensions of Grüss' inequality and its applications, Nihonkai Math. J. 13 (2002), no. 2, 159-166.

[4] D. S. Mitrinović, J. E. PeČArić, A. M. Fink, Classical and new inequalities in analysis, Mathematics and its Applications (East European Series), 61, Kluwer Academic Publishers Group, Dordrecht, 1993.

[5] Josip E. PeČarić, Frank Proschan, Y. L. Tong, Convex functions, partial orderings, and statistical applications, Mathematics in Science and Engineering, 187, Academic Press, Inc., Boston, MA, 1992.

[6] J. PEČARIĆ, B. TEPEŠ, Improvements of some inequalities for moments of guessing function, Math. Inequal. Appl. 8 (2005), no. 1, 53-62. 